\title{
A dosimetric comparison of 3D conformal vs intensity modulated vs volumetric arc radiation therapy for muscle invasive bladder cancer
}

\author{
Farshad Foroudi ${ }^{*}$, Lesley Wilson ${ }^{2}$, Mathias Bressel ${ }^{3}$, Annette Haworth ${ }^{4}$, Colin Hornby ${ }^{2}$, Daniel Pham², Jim Cramb ${ }^{4}$,
} Suki Gill', Keen Hun Tai ${ }^{1}$ and Tomas Kron ${ }^{4}$

\begin{abstract}
Background: To compare 3 Dimensional Conformal radiotherapy (3D-CRT) with Intensity Modulated Radiotherapy (IMRT) with Volumetric-Modulated Arc Therapy (VMAT) for bladder cancer.

Methods: Radiotherapy plans for 15 patients with T2-T4NOMO bladder cancer were prospectively developed for 3-DCRT, IMRT and VMAT using Varian Eclipse planning system. The same radiation therapist carried out all planning and the same clinical dosimetric constraints were used. 10 of the patients with well localised tumours had a simultaneous infield boost (SIB) of the primary tumour planned for both IMRT and VMAT. Tumour control probabilities and normal tissue complication probabilities were calculated.

Results: Mean planning time for 3D-CRT, IMRT and VMAT was 30.0, 49.3, and 141.0 minutes respectively. The mean PTV conformity (CI) index for 3D-CRT was 1.32, for IMRT 1.05, and for VMAT 1.05. The PTV Homogeneity (HI) index was 0.080 for 3D-CRT, 0.073 for IMRT and 0.086 for VMAT. Tumour control and normal tissue complication probabilities were similar for 3D-CRT, IMRT and VMAT. The mean monitor units were 267 (range 250-293) for 3D-CRT; 824 (range 641-1083) for IMRT; and 403 (range 333-489) for VMAT ( $<<0.05$ ). Average treatment delivery time were 2:25min (range 2:01-3:09) for 3D-CRT; 4:39 (range 3:41-6:40) for IMRT; and 1:14 (range 1:13-1:14) for VMAT. In selected patients, the SIB did not result in a higher dose to small bowel or rectum.

Conclusions: VMAT is associated with similar dosimetric advantages as IMRT over 3D-CRT for muscle invasive bladder cancer. VMAT is associated with faster delivery times and less number of mean monitor units than IMRT. SIB is feasible in selected patients with localized tumours.
\end{abstract}

Keywords: Bladder cancer, Intensity modulated radiation therapy, Volumetric modulated arc therapy

\section{Introduction}

Three-dimensional conformal radiotherapy (3D-CRT) has historically been the standard modality for external beam radiotherapy (RT) for bladder cancer. The morbidity of bladder cancer treated with conventional radiotherapy is well known with a RTOG update showing that $7 \%$ of their patients experienced late grade $3+$ pelvic toxicity [1]. Intensity modulated radiation therapy (IMRT) can treat less of the surrounding normal tissues

\footnotetext{
* Correspondence: farshad.foroudi@petermac.org

'Division of Radiation Oncology, Peter MacCallum Cancer Center, Peter MacCallum Cancer Institute, St Andrews Place, East Melbourne, VIC 3002, Australia

Full list of author information is available at the end of the article
}

potentially reducing normal tissue side effects. Hsieh et al. [2] found that IMRT provided good locoregional progression free survival particularly in the elderly bladder cancer group. Another advantage of IMRT is the ability to deliver more than one dose level to target volumes at the same time. This provides for the potential to deliver a simultaneous in-field boost to well localised primary sites of the tumor $[3,4]$. However, the potential negative of IMRT include the increased time required for RT delivery and the associated risk of bladder filling and changes in bladder shape and size. The magnitude of bladder filling during treatment delivery has recently been demonstrated to be approximately $1 \mathrm{~cm}^{3}$ per minute, but with wide inter patient variation

\section{Ciomed Central}


[5]. It would be particularly problematic if highly conformal dose distributions are used which are typical for IMRT. Another disadvantage of IMRT is the increased number of monitor units (MU) needed, which results in a greater integral body dose, with a probable increased risk of second malignancies [6].

VMAT is a novel type of IMRT which may be able to address both the shortcomings of IMRT discussed above. In VMAT, gantry speed, multileaf collimator (MLC) leaf position and dose rate are dynamically varied during rotation of the gantry yielding a fast and highly conformal treatment delivery [7]. While many reports on the clinical use of VMAT in the context of prostate, cervix and head and neck cancer exist [8-12] there is to our knowledge no report on the suitability of VMAT for radiotherapy of bladder cancer.

RapidArc (Varian Medical Systems, Palo Alto, CA) utilises the optimisation algorithm first described by Otto, $\mathrm{K}$ [13] to plan VMAT. This technology has recently been implemented clinically and is becoming more widely available in radiotherapy centres. Planning studies comparing VMAT with IMRT for the primary treatment of prostate cancer have demonstrated some improvements in plan quality $[14,15]$. Compared with IMRT, the potential advantages of VMAT include a large reduction in the number of MUs, with an associated reduction in treatment time, required to deliver a given fraction size.

We sought to determine if the dosimetric parameters for 3D-CRT, IMRT and VMAT for muscle invasive bladder cancer can be similar. If dosimetric outcome was similar we aimed to compare monitor units (MU) and required beam delivery time as well as planning time required for three modalities. We also aimed to compare normal tissue complication probability and tumour control probabilities between the modalities. Finally in patients with localised tumours amenable to a boost we examined the feasibility of a simultaneous infield boost (SIB) of the primary tumour for the IMRT and VMAT techniques.

\section{Method}

All 15 participant images were from a human ethics approved bladder cancer protocol, full details of this protocol have been previously published [16]. Patients underwent $\mathrm{CT}$ simulation using $3 \mathrm{~mm}$ thick slices by $3 \mathrm{~mm}$ spacing, after emptying their bladder completely. Immobilisation was with ankle stocks and a bolster under the knees. A single radiation oncologist (FF) completed contouring of the bladder, primary tumour, rectum, and non-rectal bowel prior to commencement of the project. All 3D-CRT, IMRT and VMAT plans were created prospectively using the unique set of contours for each patient by one experienced radiation therapist investigator (LW). A standalone Eclipse (Varian, Palo
Alto, CA) treatment planning system, installed on a Dell Precision T5500 computer, was used for all plan creation. The radiation therapist had extensive experience with 3D-CRT and IMRT planning, as well as training and although lesser experienced with VMAT planning was supported by a very experienced VMAT planner to mitigate any bias in recorded planning times.

The treatment plans were based on whole bladder treatment with a single phase treatment. The study was based on the original planning $\mathrm{CT}$ and contoured volumes in each of the 15 patients. The CTV consisted of the gross tumour volume (GTV) and the whole bladder. As outlined in Australian and New Zealand consensus guidelines for bladder cancer radiotherapy [17], pelvic lymph nodes were not an elective part of the volume in any patient. The CTV to PTV margin was $1.5 \mathrm{~cm}$. No oral or intravenous contrast was used at simulation. The rectum and non rectal bowel (all bowel not considered part of the rectum) were contoured 4 slices $(12 \mathrm{~mm})$ above and below the PTV. Each patient had one 3D CRT, one IMRT and one VMAT plan created by the same radiation therapist (LW). The same dose constraints were used for creation of 3D CRT, IMRT and VMAT plans (Table 1).

Table 1 Clinical Dosimetric Constraints used in Planning

\begin{tabular}{|c|c|}
\hline & 3D-CRT/IMRT/VMAT \\
\hline \multirow[t]{3}{*}{$\overline{\text { PTV }}$} & $\mathrm{D} 2 \% \geq 60.8 \mathrm{~Gy}$ \\
\hline & $\mathrm{D} 98 \% \leq 68.5 \mathrm{~Gy}$ \\
\hline & $\begin{array}{l}\text { Median absorbed dose (D } 50 \% \text { ) to be } \pm 2 \% \\
\text { of } 64 \mathrm{~Gy} \text { in } 32 \text { fractions of } 2 \mathrm{~Gy} \text { each }\end{array}$ \\
\hline \multirow[t]{4}{*}{ Bladder } & $\mathrm{V} 15 \leq 80 \%$ \\
\hline & $V 25 \leq 75 \%$ \\
\hline & V35 $\leq 70 \%$ \\
\hline & $V 50 \leq 65 \%$ \\
\hline \multirow[t]{7}{*}{ Rectum } & $\mathrm{D} 50 \leq 50 \mathrm{~Gy}$ \\
\hline & $\mathrm{D} 40 \leq 64 \mathrm{~Gy}$ \\
\hline & $\mathrm{V} 50<50 \%$ \\
\hline & $\mathrm{V} 60<35 \%$ \\
\hline & V65 < 25\% \\
\hline & V70 $<20 \%$ \\
\hline & V75 <15\% \\
\hline \multirow{3}{*}{$\begin{array}{l}\text { Rt Fem Head and } \\
\text { Lt Fem Head }\end{array}$} & $\mathrm{D} 100 \leq 35 \mathrm{~Gy}$ \\
\hline & $\mathrm{D} 60 \leq 45 \mathrm{~Gy}$ \\
\hline & $\mathrm{D} 10 \leq 50 \mathrm{~Gy}$ \\
\hline \multirow[t]{5}{*}{ Small bowel (non rectal) } & V15Gy $\leq 350 c c$ \\
\hline & V30Gy $\leq 150 c c$ \\
\hline & V35Gy $\leq 120 c c$ \\
\hline & V45Gy $\leq 50 \mathrm{cc}$ \\
\hline & V50Gy $=0 c c$ \\
\hline
\end{tabular}


The total prescription dose to the PTV was based on a median dose of 64Gy delivered in 32 fractions at $2 \mathrm{~Gy}$ per fraction treating daily, five days per week over six and the half weeks. Plans were normalised to ensure that the 95\% isodose adequately covered the PTV and that the dose distribution was such that the minimum dose to $99 \%$ of the PTV $\left(D_{99 \%}\right)$ was greater than or equal to $95 \%$ of the prescribed dose (60.8Gy) and the mean CTV dose was within $0.5 \mathrm{~Gy}$ of the prescribed dose. A number of dosimetry parameters were reported using the recommendations of the ICRU Report 83 and ASTRO to compare the treatment plans [18,19]. All plans were evaluated to ensure they met our institutional dose constraints outlined in Table 1. All 3DCRT plans were composed of a 5 beam field arrangement utilising $18 \mathrm{MV}$ photons. MLC beam shaping and beam modifiers (eg wedges) were employed as required to produce the most conformal dosimetry. All IMRT and VMAT plans were created using a $6 \mathrm{MV}$ photon beam applicable to a Varian Clinac iX (Varian Medical Systems, Palo Alto, CA) linear accelerator with a 120 leaf Millennium dynamic multileaf collimator (MLC). 6MV photons were utilised for IMRT and VMAT as this reflected most published clinical studies as well as current practice in our institution.

\section{Simultaneous infield boost (SIB)}

10 of the 15 patients whom had well localised tumours were considered for a SIB. Patients with multiple tumours were excluded from SIB planning. For the SIB the bladder tumour/site of disease was contoured as CTV2 and a $2 \mathrm{~cm}$ margin used to create PTV2. The SIB dose was an additional 10Gy, based on a modelling study by Wright et al. [20], resulting to a total dose of 74Gy in 32 fractions.

\section{IMRT plans}

IMRT plans were generated on the Eclipse Version 8.9.08 treatment planning software (Varian Medical Systems, Palo Alto, CA) using a 7 beam multifield technique. The initial optimisation parameters and their priorities were set according to our institutional optimisation protocol and then adjusted as required to achieve the dose constraints (Table 1 ) using a minimum of 60 iterations. A Normal Tissue Objective was included and the default smoothing parameters were applied to help reduce hotspots outside the PTV and the total monitor units.

\section{VMAT plans}

VMAT plans utilising the Varian RapidArc technique (Varian Medical Systems, Palo Alto, CA) were planned using Eclipse Version 8.9.08 treatment planning software using the same CT-dataset and contoured volumes as the IMRT plans. A single arc technique was used with the gantry set to rotate through $340^{\circ}$ in a clockwise direction from a starting position of $190^{\circ}$ to a final position of $170^{\circ}$ to reduce the amount of treatment through the rectum and central rail of the couch top. The collimator rotation was individually optimised for each patient but generally set at $45^{\circ}$ to reduce the effect of tongue and groove leakage.

The final dose calculation for all 3 of the plans for each patient was performed using the anisotropic analytical algorithm (AAA) version 8.9 .08 with a $2.5 \mathrm{~mm}$ dose calculation grid space.

\section{Delivery times}

Treatment was delivered for each of the 15 3D-CRT, IMRT and VMAT plans using a Varian 21iX Linear Accelerator (Varian Medical Systems, Palo Alto, CA). All beams were delivered to an empty bunker. Beam delivery time was measured using a stopwatch from the beginning of the first beam to the end of the last beam including all gantry movements. Beam order was optimised to simulate live treatment scheduling. As no patient was involved in this process, these treatment times do not include patient set-up time, which would be expected to be the same for all three plans.

\section{Conformality and homogeneity}

The degree of conformality of the plans was measured by the conformity index $(C I)$ which is a ratio of the volume of tissue receiving at least $95 \%$ of the prescribed dose divided by the volume of the PTV (Equation 1). A CI value closer to 1 is more conformal.

$$
C I=\frac{V_{95 \%}}{V_{P T V}}
$$

The homogeneity index $(H I)$ was also calculated and is the difference between the near-maximum and near-minimum dose normalised to the median dose (Equation 2) and measures the dose homogeneity across the PTV. A $H I$ value approaching zero indicates a more homogenous dose distribution within the PTV.

$$
H I=\frac{D_{2 \%}-D_{98 \%}}{D_{50 \%}}
$$

The dose was normalised to a selected percentage to give $95 \%$ of the dose to $99 \%$ of the PT. Once each plan was normalised to ensure the PTV dose reflected the prescribed dose, the DVH data was exported as a CSV file with a resolution of $0.1 \mathrm{~Gy}$ via the export functions embedded in Eclipse for importing into the TCP/NTCP software.

As the definition of $\mathrm{CI}$ does not take into account the location and the shape of the 95\% isodose volume (V95) relative to the PTV, we also calculated the Paddick's 
index which takes into account the coverage of the target volume with the 95\% isodose. The Paddick's definition of conformity index is defined as [21],

$$
\text { Paddick Index }=\frac{T V 95^{\wedge} 2}{V 95 * T V}
$$

where TV95 is the target volume (TV) within the 95\% isodose volume (V95).

\section{Radiobiology modelling}

Tumour control probability (TCP) and normal tissue complication probabilities (NTCP) were calculated using the equivalent uniform dose (EUD) mathematical models and software code described by Gay and Niemierko [22]. Briefly, these models determine EUD from the dose volume histogram (DVH) and apply a parameter ' $a$ ' which is specific to the tumour or organ at risk. The parameter $a$ can force the EUD to represent maximal, minimal or average dose. In the case of tumours, the $a$ parameter has a large negative number so that the EUD for tumours is close to the minimal dose. For normal tissues with serial like architecture, the $a$ parameter will have a large positive number, and for normal tissues that exhibit a large volume effect, $a$ will have a small positive number. Values for the $a$ parameter used in the TCP and NTCP calculations are summarised in Table 2 and were derived from the references shown in this table, or from the values recommended by Gay and Niemierko [22] if not specified in the relevant reference. The NTCP and TCP models also require values for the $\alpha / \beta$ ratio, $\mathrm{TD}_{50}$ (the dose for $50 \%$ complication rate probability) for normal tissues, $\mathrm{TCD}_{50}$ (tumour dose to control $50 \%$ of tumours irradiated), and $\gamma_{50}$ (slope of the dose response curve). For $\gamma_{50}$, we have assumed a value of 4 for late effects and 2 for the PTV unless an alternative value has been suggested by the associated reference.

\section{Statistical analysis}

The 3 treatment techniques were compared using Friedman's test. When the p-value for the test was inferior to 0.05 , a pair-wise comparison was conducted to identify which treatment techniques differ using the Wilcoxon test. No correction for multiple testing was used.

Table 2 Values for the $a$ parameter used in the TCP and NTCP calculations

\begin{tabular}{lllllll}
\hline & TCD $_{\mathbf{5 0}}$ & TD $_{\mathbf{5 0}}$ & $\boldsymbol{a}$ & $\boldsymbol{V}_{\mathbf{5 0}}$ & $\mathbf{a} / \boldsymbol{\beta}$ & Reference \\
\hline Bladder & $59 \mathrm{~Gy}$ & & -13 & 2 & 13 & Wright [20] \\
Rectal late effects & & $76.9 \mathrm{~Gy}$ & 11 & 4 & 3 & Michalski [23] \\
Non-rectal bowel & $59 \mathrm{~Gy}$ & 11 & 4 & 3 & $\begin{array}{l}\text { Kavanagh [24], } \\
\text { Left \& right femurs }\end{array}$ \\
\hline
\end{tabular}

\section{Results}

Dosimetric outcome

Table 3 shows the dosimetric outcomes of the plans created using a 3D-CRT, IMRT or VMAT technique. Using a range of dosimetry parameters, we found no statistically significant difference in target volume coverage or dose to the OARs except for the femurs that achieved a lower dose for the IMRT and VMAT techniques compared with 3DCRT. With SIB the dosimetric parameters for in favour of VMAT, such as for left femur and nonrectal bowel, none of these can be considered a clinical significant difference.

\section{PTV conformity index $(\mathrm{CI})$, homogenity index $(\mathrm{HI})$, and Paddick's index}

The mean PTV conformity (CI) index for 3D-CRT was 1.32, for IMRT 1.05, and for VMAT 1.05. The PTV HI index was 0.080 for 3D-CRT, 0.073 for IMRT and 0.086 for VMAT. The Paddick index was smaller for 3D-CRT than IMRT $(-0.18, \mathrm{p}<0.001)$, and VMAT $(-0.16$, $\mathrm{p}<0.001)$. The Paddick index difference between IMRT and VMAT $(0.02, \mathrm{p}<0.015)$ is considered clinically insignificant.

\section{Planning time}

The total planning time was found to be a mean of 30.7 minutes (range 10-45 minutes) for 3D-CRT; 49.33 minutes (range 20-90 minutes) for IMRT; and 141 minutes (60-240 minutes) for VMAT. Total times on a networked Eclipse system would be shorter, since calculations are distributed across multiple workstations when using AAA algorithm. 3DCRT was significantly quicker than VMAT and IMRT $(\mathrm{P}<0.05)$ while IMRT was quicker than VMAT $(\mathrm{P}<0.05)$. There was no obvious trend in planning times (such as a decrease in time with experience) over the study period.

\section{Monitor units}

The mean monitor units was lowest for 3D-CRT at 267 (range 250-293); was 403 (range 333-489) for VMAT, and highest at 824 (range 641-1083) for IMRT; $(\mathrm{P}<0.05)$.

\section{Treatment times}

Average treatment delivery time were 2:25 min (range 2:01-3:09) for 3D-CRT; 4:39 min (range 3:41-6:40) for IMRT; and were shortest at 1:14 min (range 1:13-1:14) for VMAT.

\section{Tumour control probability and normal tissue complication probabilities}

Table 4 shows the TCP and NTCP for 3D-CRT, IMRT and VMAT. As can be seen most probabilities are similar. With the use of IMRT and VMAT the probability of femoral head complications was lower that with 3D-CRT. 
Table 3 Dosmetric values for whole bladder treatment

\begin{tabular}{|c|c|c|c|}
\hline & 3DCRT & IMRT & VMAT \\
\hline & $\overline{\text { Mean (min-max, st dev) }}$ & $\overline{\text { Mean (min-max, stdev) }}$ & Mean (min-max, stdev) \\
\hline CTV MAX & $66.8(65.1-68.4,0.94)$ & $66.1(65.5-66.7,0.41)$ & $66.7(65.7-68,0.65)$ \\
\hline CTV MIN & $63.1(62.2-63.7,0.44)$ & $62.4(61.9-63.1,0.34)$ & $61.8(61-62.6,0.49)$ \\
\hline CTV MEAN & $64.4(64.2-64.5,0.11)$ & $64.3(63.8-64.5,0.2)$ & $64.3(63.9-64.5,0.19)$ \\
\hline CTV MEDIAN & $64.2(63.9-64.4,0.15)$ & $64.4(63.7-64.6,0.24)$ & $64.4(63.9-64.6,0.2)$ \\
\hline CTV D98\% & $63.4(62.9-63.9,0.3)$ & $63(62.7-63.7,0.27)$ & $62.9(62.4-63.5,0.35)$ \\
\hline CTV D2\% & $66.1(64.8-67.5,0.74)$ & $65.6(64.8-66.2,0.4)$ & $65.6(64.9-66.1,0.36)$ \\
\hline CTV D99 > 60.8Gy & $63.3(62.8-63.8,0.32)$ & $62.9(62.6-63.6,0.28)$ & $62.7(62.2-63.4,0.39)$ \\
\hline PTV MAX & $66.9(65.3-68.5,0.9)$ & $67(66-68,0.63)$ & $68.2(67.1-69.5,0.7)$ \\
\hline PTV MIN & $58.9(56.7-60.6,1,17)$ & $54(46.5-58.1,3.68)$ & $54.4(45.2-58.6,3.16)$ \\
\hline PTV MEAN & $64.2(63.9-64.4,0.16)$ & $64.2(63.9-64.6,0.2)$ & $64.2(63.5-64.7,0.33)$ \\
\hline PTV MEDIAN & $64.2(63.9-64.4,0.17)$ & $64.4(64-64.8,0.22)$ & $64.3(63.9-64.9,0.27)$ \\
\hline PTV V95\% (cc) & $624(299-987,208)$ & $495(244-765,159)$ & $490(246-751,150)$ \\
\hline PTV D95\% & $62.3(61.6-63,0.42)$ & $62.3(61.5-62.7,0.31)$ & $61.8(59.6-62.8,0.82)$ \\
\hline PTV D2\% & $66.5(65.1-68,0.82)$ & $65.9(65.2-66.2,0.32)$ & $66.3(65.7-67,0.39)$ \\
\hline PTV D50\% & $64.2(63.9-64.4,0.17)$ & $64.4(64-64.8,0.22)$ & $64.3(63.9-64.9,0.28)$ \\
\hline PTV D99 > 60.8Gy & $61.1(60.4-62.3,0.54)$ & $60.4(59.6-61.3,0.55)$ & $60.2(57-61.6,1.11)$ \\
\hline PTV D98 > 60.8Gy & $61.6(60.9-62.6,0.51)$ & $61.2(60.4-61.8,0.36)$ & $60.8(58.2-62,0.97)$ \\
\hline Paddix Index & $0.75(0.71-0.8,0.02)$ & $0.92(0.87-0.97,0.03)$ & $0.91(0.88-0.94,0.02)$ \\
\hline RECTUM MEDIAN & $35(16.4-58.1,12)$ & $36.7(19.4-44.8,5.89)$ & $39.6(19.9-51.3,7.6)$ \\
\hline RECTUM D98\% & $9.06(1.58-24.4,8.06)$ & $6.38(2.19-16.4,4.93)$ & $7.37(2.54-19.9,5.3)$ \\
\hline RECTUM D2\% & $62.1(41.6-64.8,5.71)$ & $62.4(50.3-65.3,3.44)$ & $63.3(45-66.8,5.18)$ \\
\hline RECTUM D60 < 40Gy & $31.2(14.2-54.2,11.2)$ & $33(14.5-37.9,5.75)$ & $34.3(14.4-44.9,8)$ \\
\hline RECTUM D50 < 50Gy & $35(16.4-58.2,12)$ & $36.8(19.4-44.8,5.85)$ & $39.4(19.9-51.2,7.71)$ \\
\hline RECTUM D40 < 64Gy & $40.7(18.7-60.5,12)$ & $40.7(25.1-51.4,5.77)$ & $44.1(23.4-57.3,7.96)$ \\
\hline RT FEMUR MEDIAN & $40(36-43.2,2.8)$ & $21.5(19.5-23,1.08)$ & $17.3(13.1-21.5,2.77)$ \\
\hline RT FEMUR D98\% & $9.55(1.84-31.8,7.84)$ & $2.62(1.46-6.61,1.37)$ & $2.27(1.32-4.2,0.84)$ \\
\hline RT FEMUR D2\% & $49.9(41.2-61,4.9)$ & $34.8(25.4-51.9,6.74)$ & $35.1(28-52,5.99)$ \\
\hline RT FEMUR D100 < 35Gy & $5.24(1.32-19,4.73)$ & $1.74(1.16-3.2,0.58)$ & $1.65(0.9-2.83,0.61)$ \\
\hline RT FEMUR D60 < 45Gy & $38.5(32.6-42.3,2.97)$ & $20.6(18.5-22.2,1.24)$ & $15.4(11.5-20.5,3.14)$ \\
\hline RT FEMUR D10 < 50Gy & $46.4(39.6-50.7,3.41)$ & $28.6(23.8-37.5,3.88)$ & $29(22.6-40.8,4.23)$ \\
\hline LT FEMUR MEDIAN & $39.9(35.4-43.2,2.83)$ & $22.1(20.7-24.3,1.07)$ & $15.5(12.1-18.8,2.4)$ \\
\hline LT FEMUR D98\% & $10.4(1.6-32.1,8.66)$ & $2.79(1.32-7.38,1.71)$ & $2.51(1.16-5.12,1.12)$ \\
\hline LT FEMUR D2\% & $47.8(41.7-51.6,3.36)$ & $32.3(26.6-42.6,4.24)$ & $32.4(26.1-38.9,3.38)$ \\
\hline LT FEMUR D100 < 35Gy & $5.45(1.17-15.8,4.37)$ & $1.75(0.92-3.28,0.69)$ & $1.84(0.88-3.88,0.85)$ \\
\hline LT FEMUR D60 < 45Gy & $37.3(29.2-42.1,3.84)$ & $21.4(19.8-23.4,1.09)$ & $12.9(8.48-15.4,2.2)$ \\
\hline LT FEMUR D10 < 50Gy & $45.1(39.4-49.8,3.38)$ & $26.7(24.5-31.5,1.96)$ & $27.2(21.2-32.2,2.86)$ \\
\hline NON-RECTAL BOWEL MEDIAN & $48.9(20.6-63.2,14.2)$ & $42.2(11.3-62.9,14.5)$ & $42(10.5-63,14.3)$ \\
\hline NON-RECTAL BOWEL D98\% & $13.6(2.08-21.8,6.51)$ & $13.1(2.72-30.6,7.94)$ & $12.5(2.73-29.1,7.63)$ \\
\hline NON-RECTAL BOWEL D2\% & $65(62.1-67,1.04)$ & $65.3(64.6-66.1,0.47)$ & $65.6(64.6-66.6,0.52)$ \\
\hline NON-RECTAL BOWEL D30<40Gy & $58.7(40.4-64.2,7.06)$ & $55.6(34.2-64.9,9.65)$ & $55.6(36.5-64.5,8.94)$ \\
\hline NON-RECTAL BOWEL D0 < 45Gy & $65.9(64.3-67.8,0.88)$ & $66.1(65.3-67,0.54)$ & $66.9(65.7-67.8,0.64)$ \\
\hline SMALL BOWEL MEDIAN & $44.4(16.1-63.5,17)$ & $38(15.9-63.7,15.2)$ & $35.8(14.7-63.1,15.6)$ \\
\hline SMALL BOWEL D98\% & $18.1(2.19-55.2,14.1)$ & $13.4(3.04-29.4,8.53)$ & $12.2(3.13-27.2,7.57)$ \\
\hline
\end{tabular}


Table 3 Dosmetric values for whole bladder treatment (Continued)

\begin{tabular}{llll}
\hline SMALL BOWEL D2\% & $59.3(39-67.3,10)$ & $56.5(30-66.1,14.1)$ & $56.3(29.4-66.9,14.8)$ \\
SMALL BOWEL D30 <40Gy & $52.7(21.3-64.7,16.6)$ & $49.9(23.6-63.7,16)$ & $49.5(21.5-64.5,17.3)$ \\
SMALL BOWEL D0 < 45Gy & $62.9(49.9-67.8,5.66)$ & $60.3(33.5-67,11.5)$ & $60.4(31.5-68.4,12.8)$ \\
LARGE BOWEL MEDIAN & $48.8(9.27-62.7,14.9)$ & $42.5(7.71-64,15.1)$ & $42.8(6.58-64,14.7)$ \\
LARGE BOWEL D98\% & $13.5(1.9-20.6,6.11)$ & $12.9(2.34-30.6,7.62)$ & $12(2.26-29.1,7.26)$ \\
LARGE BOWEL D2\% & $64.9(63.3-66.5,0.92)$ & $65.1(64.3-65.8,0.48)$ & $65.3(64.1-66.2,0.65)$ \\
LARGE BOWEL D30<40Gy & $58.9(36.4-64,7.24)$ & $55.1(32.1-64.7,9.77)$ & $54.4(25.9-64.9,11.3)$ \\
LARGE BOWEL D0<45Gy & $65.5(62.9-67.5,1.12)$ & $66(65.3-66.9,0.48)$ & $66.7(65.7-68.4,0.77)$ \\
\hline
\end{tabular}

\section{Simultaneous infield boost (SIB)}

Table 5 shows the comparison of IMRT and VMAT for a SIB in the 10 patients with well demarcated tumours. VMAT was associated with a longer planning time but reduced MUs for treatment. Doses to rectum, small bowel and non rectal bowel were comparable for 3DCRT without boost, versus IMRT and VMAT with SIB. This is illustrated in Table 5.

\section{Discussion}

Minimising time taken in the treatment room is important for a variety of reasons including patient satisfaction, as well as departmental throughput. In addition, the bladder is a dynamic soft tissue organ, the size and shape can vary according to urine filling and its position can vary according to its size as well as due to rectal filling. Hence it would seem to be the ideal organ for daily online imaging with corrections based on soft tissue position $[16,27,28]$. The planning datasets in this study are all from patients treated using daily online image guided adaptive radiotherapy [16]. Intrafraction motion and bladder filling is a concern if there is a significant daily treatment duration. Keeping the treatment time as short as possible is important to reduce intrafraction filling as a recent MRI based study has shown increased filling in intervals up to 28 minutes [5]. Studies using ultrasound [29] as well as MRI [30] have shown in certain cases filling and motion during time in the treatment bunker can be significant. With changes in technology such as faster CBCT reconstruction times, increased treatment automation and volumetric arc therapy, time in the treatment bunker is reducing.

A number of studies in other tumour sites have shown that VMAT results in similar plan quality with substantially reduced treatment times compared to IMRT $[7,14,31,32]$. The combination of similar plan quality and reduced treatment time shows the clear advantage of VMAT over IMRT. In an early report of IMRT in muscle invasive bladder cancer, Budgell et al. [33] found that using a 4 field IMRT technique was feasible and took an average of 20 minutes treatment delivery time per treatment fraction. Muren et al. [34] examined concomitant tumour boost for bladder cancer using IMRT, finding such a technique feasible in 9 of 10 planned cases. It is important to note that in only $50 \%$ of cases, the dosimetric criteria were met with a 5 field plan but in $90 \%$ of cases the criteria were meet using a 7 or 9 field plan [34] at the penalty of increased low dose irradiation of surrounding normal tissues. While there has been an examination of VMAT for simultaneous integrated boost for intraprostatic lesions [35] there is little reported in the literature for bladder cancer.

In our study all contouring and planning was conducted by the same investigators prospectively. It may be possible that the longer VMAT planning times may be reduced as the staff become even more experienced in

Table 4 Tumour Control and Normal Tissue complication Probabilities

\begin{tabular}{llll}
\hline & \multicolumn{1}{l}{ 3D-CRT } & IMRT & \multicolumn{1}{c}{ VMAT } \\
\cline { 2 - 4 } & Mean(min-max, st dev) & Mean(min-max, st dev) & $63.9(62.6-64.5,0.49)$ \\
\hline EUD PTV & $64(63.7-64.3,0.19)$ & $64(63.7-64.5,0.23)$ & $65 \%(62 \%-67 \%, 1 \%)$ \\
TCP PTV & $66 \%(65 \%-67 \%, 1 \%)$ & $66 \%(65 \%-67 \%, 1 \%)$ & $52.5(37.2-59.2,4.94)$ \\
EUD rectum & $52.9(36.1-58,5.04)$ & $51.1(36.8-57.7,4.63)$ & $24.3(18.4-40.3,5.88)$ \\
EUD RTFEM & $38.9(31.2-47.8,4.29)$ & $24.9(16.6-41.8,6.51)$ & $57.3(50.7-61.6,2.95)$ \\
EUD NRB & $57.8(49.4-62.1,3.18)$ & $57.2(50.2-61.6,2.93)$ & $40 \%(8 \%-67 \%, 17 \%)$ \\
NTCP NRB & $44 \%(5 \%-69 \%, 18 \%)$ & $40 \%(7 \%-67 \%, 17 \%)$ & $21.7(16.6-28,2.99)$ \\
EUD LFH & $37.1(31-41.2,3.23)$ & $22.9(17.6-29.2,3.46)$ & \\
\hline
\end{tabular}


Table 5 SIB comparison using IMRT and VMAT

\begin{tabular}{|c|c|c|c|}
\hline \multirow[t]{2}{*}{ Variable } & \multirow{2}{*}{$\begin{array}{l}\text { IMRT BOOST } \\
\text { Mean(min-max, st dev) }\end{array}$} & \multirow{2}{*}{$\frac{\text { VMAT BOOST }}{\text { Mean(min-max, st dev) }}$} & \multirow[b]{2}{*}{$\mathrm{p}$-value } \\
\hline & & & \\
\hline CTV MAX & $76.1(75.1-77.2,0.69)$ & $77.4(76-78.4,0.66)$ & 0.004 \\
\hline CTV MIN & $63.2(61.7-66.3,1.33)$ & $61.5(60.2-63.5,1)$ & 0.011 \\
\hline CTV MEAN & 71.5(68.7- 74.3, 1.75) & $71.2(68.6-73.2,1.43)$ & 0.398 \\
\hline CTV MEDIAN & $72.3(67-74.6,2.88)$ & $71.9(66.9-74.1,2.62)$ & 0.068 \\
\hline CTV D98\% & $64.8(62.8-69.7,2.2)$ & $64.4(62.9-68,1.73)$ & 0.625 \\
\hline CTV D2\% & 75.4(74.6-76.4-0.55) & $76(75-76.6,0.48)$ & 0.007 \\
\hline CTV D99 > 60.8Gy & $64.5(62.5-69.1,2.08)$ & $63.8(62.4-69.1,1.85)$ & 0.044 \\
\hline PTV MAX & $76.7(75.1-78.3,0.9)$ & $78.3(76.1-80.2,1.15)$ & 0.006 \\
\hline PTV MIN & $55.1(47.3-57.4,2.9)$ & $55.5(15.5-57.6,1.93)$ & 0.476 \\
\hline PTV MEAN & $68.8(67.5-70.4,1.2)$ & $68.8(67-70.4,0.89)$ & 1.000 \\
\hline PTV MEDIAN & $67.9(65.5-71.6,2.58)$ & 67.7(65.9. 70.1, 1.37) & 0.688 \\
\hline PTV V95\% (cc) & $557(254-798,171)$ & $552(267-804,173)$ & 0.756 \\
\hline PTV D95\% & $63.1(62.5-63.7,0.46)$ & $62.9(62-63.9,0.56)$ & 0.450 \\
\hline PTV D2\% & $75.5(74.5-76.3,0.49)$ & $75.9(73.4-76.8,0.96)$ & 0.056 \\
\hline PTV D50\% & $68.2(65.8-71.6,2.45)$ & $67.7(65.9-70.1,1.37)$ & 0.350 \\
\hline PTV D99 > 60.8Gy & $61.5(60.5-62.5,0.58)$ & $61.9(60.3-70,2.73)$ & 0.307 \\
\hline PTV D98 > 60.8Gy & $62.2(61.5-62.9,0.47)$ & $61.9(61-63,0.63)$ & 0.307 \\
\hline BOOST GTV MAX & $75.7(74.9-76.9,0.57)$ & 76.6(75.4-77.6, 0.74) & 0.006 \\
\hline BOOST GTV MIN & $73.2(72.3-74,0.52)$ & $72.9(71-77.2,1.58)$ & 0.142 \\
\hline BOOST GTV MEAN & $74.5(74.4-74.5,0.02)$ & $74.5(74.4-74.5,0.03)$ & 0.357 \\
\hline BOOST GTV MEDIAN & $74.5(74.2-74.6,0.1)$ & $74.5(74.4-74.6,0.04)$ & 0.308 \\
\hline BOOST GTV D98\% & $73.5(72.9-74.2,0.45)$ & $73.2(72.5-73.8,0.39)$ & 0.075 \\
\hline BOOST GTV D2\% & $75.3(74.8-76,0.38)$ & $75.7(75.1-76.5,0.46)$ & 0.029 \\
\hline BOOST GTV D99 > 70.30Gy & $73.5(72.7-74.2,0.49)$ & $73.1(72.2-73.3,0.46)$ & 0.068 \\
\hline BOOST PTV MAX & $76.6(75.1-78.3,0.9)$ & $78.6(77-80.2,0.9)$ & 0.004 \\
\hline BOOST PTV MIN & $66.4(63.3-69.5,1.9)$ & $67.1(65.2-69.5,1.45)$ & 0.168 \\
\hline BOOST PTV MEAN & $74.2(73.6-74.5,0.26)$ & $74.1(73.7-74.4,0.22)$ & 0.056 \\
\hline BOOST PTV MEDIAN & $74.4(73.8-74.6,0.28)$ & $74.3(74-74.6,0.17)$ & 0.350 \\
\hline BOOST PTV V95\% (cc) & $193(60.8-273,65.1)$ & $179(61.3-250,55.9)$ & 0.083 \\
\hline BOOST PTV D95\% & $71.9(71-72.9,0.65)$ & $71.3(70.2-72.2,0.6)$ & 0.023 \\
\hline BOOST PTV D2\% & 75.8(74.7-76.6, 0.53) & $76.7(75.8-77.5,0.48)$ & 0.004 \\
\hline BOOST PTV D50\% & 74.4(73.8-74.6, 0.28) & $74.3(74-74.6,0.17)$ & 0.350 \\
\hline BOOST PTV D99 > 70.30Gy & $70.4(68-72,1.08)$ & $70.3(69.3-72,0.78)$ & 0.683 \\
\hline BOOST PTV D98 > 70.30Gy & $71.1(69.9-72.4,0.81)$ & $70.7(69.7-72.4,0.75)$ & 0.185 \\
\hline RECTUM MEDIAN & $36.4(19.2-43.9,6.31)$ & $37.1(19.2-49.1,8.02)$ & 0.351 \\
\hline RECTUM D98\% & $5.36(2.28-15.4,4.3)$ & $6.3(2.62-19.5,4.78)$ & 0.045 \\
\hline RECTUM D2\% & $66.1(50.8-72.6,6.18)$ & $66.8(44-75,8.44)$ & 0.100 \\
\hline RECTUM D60 < 40Gy & $32.5(14.5-38,6.39)$ & $32.5(14.9-41.9,7.6)$ & 0.423 \\
\hline RECTUM D50 < 50Gy & $36.4(19.2-43.9,6.32)$ & $37.1(19.2-49.1,8)$ & 0.415 \\
\hline RECTUM D40 < 64Gy & $41(24-52.5,6.9)$ & $41.8(22.7-55.9,8.68)$ & 0.398 \\
\hline RT FEMUR MEDIAN & $24(19.5-27.9,2.23)$ & $23.9(16.6-31.8,4.28)$ & 0.965 \\
\hline RT FEMUR D98\% & $3.07(1.73-6.82,1.54)$ & $2.86(1.18-5.45,1.09)$ & 0.625 \\
\hline RT FEMUR D2\% & $39.5(30.7-54.8,7.3)$ & $42.4(33-55,5.96)$ & 0.142 \\
\hline
\end{tabular}


Table 5 SIB comparison using IMRT and VMAT (Continued)

\begin{tabular}{|c|c|c|c|}
\hline RT FEMUR D100 < 35Gy & $1.96(1.17-3.33,0.65)$ & $2.1(1.28-3.96,0.75)$ & 0.126 \\
\hline RT FEMUR D60 < 45Gy & $22.9(17.5-27.2,2.59)$ & $22.8(14.4-34.5,5.6)$ & 0.760 \\
\hline RT FEMUR D10<50Gy & $31.8(27.1-39,3.83)$ & $36.8(29.9-47.4,4.86)$ & 0.041 \\
\hline LT FEMUR MEDIAN & $24(19.2-28,2.27)$ & $20(16.2-24.6,2.31)$ & 0.009 \\
\hline LT FEMUR D98\% & $3.18(1.73-7.74,1.87)$ & $3.19(1.81-5.81,1.35)$ & 0.213 \\
\hline LT FEMUR D2\% & $35.5(29.6-46.9,5.33)$ & $37.4(30.1-45.5,5.57)$ & 0.505 \\
\hline LT FEMUR D100 < 35Gy & $1.96(1.2-3.33,0.62)$ & $2.27(1.28-3.96,0.91)$ & 0.044 \\
\hline LT FEMUR D60 < 45Gy & 23.1(17.9-27.1, 2.35) & $18.5(14.8-24.1,2.66)$ & 0.008 \\
\hline LT FEMUR D10<50Gy & $29.2(27-32.9,2.13)$ & $32.7(27.4-40.2,4.7)$ & 0.053 \\
\hline NON-RECTAL BOWEL MEDIAN & $48.3(29.5-65.8,11.6)$ & $46.3(28.7-63.6,12.1)$ & 0.029 \\
\hline NON-RECTAL BOWEL D98\% & $15.6(7.5-26.9,7.38)$ & $13.5(6.63-31.9,7.86)$ & 0.045 \\
\hline NON-RECTAL BOWEL D2\% & $72.3(66.8-75.5,3.13)$ & $72.3(67-76.1,3.37)$ & 0.894 \\
\hline NON-RECTAL BOWEL D30 < 40Gy & 59.2(33.6-71.7, 9.99) & $58.4(37.2-68.8,9.38)$ & 0.351 \\
\hline NON-RECTAL BOWEL D0 < 45Gy & 75.3(71.5-77.1, 1.53) & 76.2(71.6-78.7, 2.19) & 0.023 \\
\hline SMALL BOWEL MEDIAN & $42.5(18.3-65.7,14.8)$ & $37.5(14.4-63.5,16.6)$ & 0.019 \\
\hline SMALL BOWEL D98\% & 16.7(5.81-33.6, 9.95) & $12.7(5.88-25.7,6.73)$ & 0.008 \\
\hline SMALL BOWEL D2\% & $62(37-75.8,16.4)$ & $59.5(28-77.2,20.4)$ & 0.221 \\
\hline SMALL BOWEL D30<40Gy & $50.9(23.3-70.5,17.1)$ & $47.2(17.3-67.2,20.4)$ & 0.076 \\
\hline SMALL BOWEL D0 < 45Gy & $68.8(43.5-76.3,13)$ & $67.6(30-78.7,17.5)$ & 1.000 \\
\hline LARGE BOWEL MEDIAN & $50.7(28.4-67,11.1)$ & $48.5(25.4-65,12.2)$ & 0.023 \\
\hline LARGE BOWEL D98\% & $15.1(6.46-26.9,6.71)$ & $13.6(6.23-31.9,7.34)$ & 0.068 \\
\hline LARGE BOWEL D2\% & $71.3(65.3-75.7,3.29)$ & $71.6(66.8-76.3,3.19)$ & 0.286 \\
\hline LARGE BOWEL D30 < 40Gy & $61.7(45.5-73.3,6.52)$ & $60.2(37.8-72.9,8.91)$ & 0.168 \\
\hline LARGE BOWEL D0 < 45Gy & $74.8(71.5-77.1,1.52)$ & 75.4(71.6-78.6, 2.3) & 0.142 \\
\hline PLANNING TIME (MINS) & $65.5(45-100,19.7)$ & $159(60-250,54.3)$ & 0.005 \\
\hline TOTAL MU & $822(684-1047,94.1)$ & 419(359-464, 34.4) & 0.004 \\
\hline PTV CONFORMITY INDEX (CI) & $1.12(1.04-1.29,0.07)$ & $1.11(1.05-1.22,0.05)$ & 0.953 \\
\hline PTV BOOST CONFORMITY INDEX (CI) & $1.14(0.99-1.48,0.16)$ & $1.06(0.97-1.23,0.08)$ & 0.068 \\
\hline PTV HOMOGENITY INDEX (HI) & $0.196(0.18-0.22,0.014)$ & $0.2(0.08-0.24,0.04)$ & 0.258 \\
\hline PTV BOOST HOMOGENITY INDEX (HI) & $0.06(0.03-0.08,0.02)$ & $0.081(0.05-0.1,0.014)$ & 0.009 \\
\hline EUD PTV & $67.3(65.8-69,1.24)$ & $67.3(66-69.1,0.8)$ & 0.965 \\
\hline TCP PTV & $74 \%(71 \%-78 \%, 3 \%)$ & $74 \%(71 \%-78 \%, 2 \%)$ & 0.965 \\
\hline EUD PTV2 & $66(64.6-67.7,1.21)$ & $66(64.7-67.7,0.78)$ & 0.965 \\
\hline TCP PTV2 & 71\%(67\%-75\%, 3\%) & $71 \%(68 \%-75 \%, 2 \%)$ & 0.965 \\
\hline EUD rectum & $54.1(37.1-61.6,6.71)$ & $55.6(38.7-64.4,7.11)$ & 0.011 \\
\hline EUD RTFEM & $29.4(21.1-43.7,6.42)$ & $30.6(22.1-42.9,5.8)$ & 0.505 \\
\hline EUD NRB & $62.6(52.4-72.1,5.05)$ & $62.6(52.8-71,4.95)$ & 0.894 \\
\hline NTCP NRB & 68\%(13\%-96\%, 23\%) & 67\%(14\%-95\%, 23\% & 0.722 \\
\hline EUD LFH & $24.5(19.8-32.4,3.81)$ & $25.9(19.6-33.2,4.89)$ & 0.450 \\
\hline
\end{tabular}

this newer technology. However we did not find a trend of planning times decreasing over the study period.

While our study did not specifically examine radiation-induced second malignancies, they are an infrequent but feared complication of treatment [36].
IMRT leads to an increase in monitor units compared to 3D-CRT and therefore is likely to increase the integral dose [6]. Ruben et al. found that the effect on secondary cancer induction by spreading out the low to intermediate dose with IMRT is small [37]. While IMRT increases 
the MU demand compared to 3D-CRT, the smaller field size and reduced average filed intensity have been reported to reduce the scatter more than sufficiently to compensate for any increase in head leakage [37]. The decrease in MUs required with VMAT reduces exposure to leaked radiation from the gantry head, which is a concern regarding the development of second cancers [38]. However VMAT delivers dose circumferentially around patients, potentially leading to an increase in the volume of tissue exposed to low radiation doses. While we found that the monitor units with VMAT was significantly lower than for IMRT, as both the delivered dose distribution and leakage radiation play a role in depositing dose outside the treatment volume, the potential for secondary malignancies after curative treatment remains uncertain across these two techniques [10].

Reduced MUs does have other advantages in the running of radiotherapy departments including extended linear accelerator lifespan, reduced shielding requirements as well as the likely economic benefit of faster treatment and throughput. The reduction in treatment times with use of VMAT are particularly useful for bladder cancer treatment given the organ can fill with urine and move with increasing treatment times. The results of our study for VMAT are promising, but only a randomised clinical study can show the real effects of the introduction of such technique. Unfortunately, with low levels of referral for organ preservation for bladder cancer in many countries, such randomised studies remain unlikely.

\section{Conclusions}

Radiotherapy to the bladder is complicated by the fact that bladder volume may increase during delivery. If bladder cancer patients are to benefit from modern radiotherapy such as IMRT, it is essential to deliver the treatment as fast as possible. We were able to demonstrate that IMRT and VMAT plans are similar in terms of dose distributions and both have superior conformity indices when compared to 3DCRT. Given the benefits in terms of reduced MU as well as treatment time, VMAT appears to be the ideal technology to be used with daily image guided or adaptive radiotherapy for muscle invasive bladder cancer.

\section{Competing interests}

The authors declare that they have no competing interests.

\section{Authors' contributions}

FF devised the study protocol, carried out all the contouring, wrote the manuscript draft and had overall responsibility for the study. LW carried all the 3D-CRT, IMRT and VMAT plans as well as providing input into the manuscript. MB provided statistical input into the protocol and carried out all the statistical analysis, as well as input into the manuscript. AH advised regarding the study protocol, carried all the radiobiological modelling and provided input into the final manuscript. $\mathrm{CH}$ provided radiation therapy input into protocol, advice during the planning process as well as input into the final manuscript. DP provided input into study design. Transferred all datasets for planning, carried out all treatment timings as well as input into the manuscript. JC provided medical physics input into the study design, conduct as well as the manuscript. SC provided input into the study design, protocol text and manuscript text KHT provided input into the study design, protocol text and manuscript text. TK provided overall medical physics input and advice into the protocol, study conduct and manuscript. All authors read and approved the final manuscript.

\section{Acknowledgements}

Partly funded by a NHMRC Project Grant. Note Peter MacCallum Cancer Centre and investigators have a Varian Medical Systems research collaborative agreement.

\section{Author details}

'Division of Radiation Oncology, Peter MacCallum Cancer Center, Peter MacCallum Cancer Institute, St Andrews Place, East Melbourne, VIC 3002, Australia. ${ }^{2}$ Radiation Therapy Services, Peter MacCallum Cancer Center, Melbourne, VIC, Australia. ${ }^{3}$ Biostatistics and Clinical Trials, Peter MacCallum Cancer Centre, Melbourne, VIC, Australia. ${ }^{4}$ Physical Sciences, Peter MacCallum Cancer Center, Melbourne, VIC, Australia.

Received: 15 March 2012 Accepted: 3 July 2012

Published: 23 July 2012

\section{References}

1. Efstathiou JA, Bae K, Shipley WU, et al: Late pelvic toxicity after bladdersparing therapy in patients with invasive bladder cancer: RTOG 89-03, 95-06, 97-06, 99-06. J Clin Oncol 2009, 27:4055-61.

2. Hsieh $\mathrm{CH}$, Chung SD, Chan PH, et al: Intensity modulated radiotherapy for elderly bladder cancer patients. Radiat Oncol 2011, 6:75.

3. Cilla S, Caravatta L, Picardi V: et al. Clin Oncol (R Coll Radiol): Volumetric Modulated Arc Therapy with Simultaneous Integrated Boost for Locally Advanced Rectal Cancer; 2011.

4. Lee TF, Ting HM, Chao PJ, Fang FM: Dual Arc Volumetric-modulated Arc Radiotherapy (VMAT) of Nasopharyngeal Carcinomas: A Simultaneous Integrated Boost Treatment Plan Comparison with Intensity-modulated Radiotherapies and Single Arc VMAT. Clin Oncol (R Coll Radiol) 2011, 24:196-207.

5. McBain C, Khoo V, Buckley D, et al: Assessment of bladder motion for clinical radiotherapy practice using cine-magnetic resonance imaging Int J Radiat Oncol Biol Phys 2009, 75:664-671.

6. Hall EJ, Wuu CS: Radiation-induced second cancers: the impact of 3D-CRT and IMRT. Int J Radiat Oncol Biol Phys 2003, 56:83-88.

7. Guckenberger M, Richter A, Krieger T, Wilbert J, Baier K, Flentje M: Is a single arc sufficient in volumetric-modulated arc therapy (VMAT) for complex-shaped target volumes? Radiother Oncol 2009, 93:259-265.

8. Cozzi L, Dinshaw KA, Shrivastava SK, et al: A treatment planning study comparing volumetric arc modulation with RapidArc and fixed field IMRT for cervix uteri radiotherapy. Radiother Oncol 2008, 89:180-191.

9. Alvarez-Moret J, Pohl F, Koelbl O, Dobler B: Evaluation of volumetric modulated arc therapy (VMAT) with Oncentra MasterPlan(R) for the treatment of head and neck cancer. Radiat Oncol 2010, 5:110.

10. Davidson MT, Blake SJ, Batchelar DL, Cheung P, Mah K: Assessing the role of volumetric modulated arc therapy (VMAT) relative to IMRT and helical tomotherapy in the management of localized, locally advanced, and post-operative prostate cancer. Int I Radiat Oncol Biol Phys 2011, 80:1550-1558

11. Tsai CL, Wu JK, Chao HL, Tsai YC, Cheng JC: Treatment and Dosimetric Advantages Between Vmat, Imrt, and Helical Tomotherapy in Prostate Cancer. Med Dosim 2010

12. Wolff $D$, Stieler F, Welzel G, et al: Volumetric modulated arc therapy (VMAT) vs. serial tomotherapy, step-and-shoot IMRT and 3D-conformal RT for treatment of prostate cancer. Radiother Oncol 2009, 93:226-233.

13. Otto K: Volumetric modulated arc therapy: IMRT in a single gantry arc Med Phys 2008, 35:310-317.

14. Palma D, Vollans $E$, James $K$, et al: Volumetric modulated arc therapy for delivery of prostate radiotherapy: comparison with intensity-modulated radiotherapy and three-dimensional conformal radiotherapy. Int J Radiat Oncol Biol Phys 2008, 72:996-1001. 
15. Hardcastle N, Tome WA, Foo K, Miller A, Carolan M, Metcalfe P: Comparison of prostate imrt and vmat biologically optimised treatment plans. Med Dosim 2010, 36:264-271.

16. Foroudi F, Wong J, Kron T, et al: Online Adaptive Radiotherapy for MuscleInvasive Bladder Cancer: Results of a Pilot Study. Int J Radiat Oncol Biol Phys 2010, 81:765-771.

17. Hindson BR, Turner SL, Millar JL, et al: Australian \& New Zealand Faculty of Radiation Oncology Genito-Urinary Group: 2011 consensus guidelines for curative radiotherapy for urothelial carcinoma of the bladder. $J$ Med Imaging Radiat Oncol 2012, 56:18-30.

18. Holmes T, Das R, Low D, et al: American Society of Radiation Oncology recommendations for documenting intensity-modulated radiation therapy treatments. Int J Radiat Oncol Biol Phys 2009, 74:1311-1318.

19. (ICRU) ICORUaM: ICRU Report 83, Prescribing, Recording, and Reporting Intensity-Modulated Photon-Beam Therapy (IMRT). Bethesda: ICRU; 2010. 2010.

20. Wright $P$, Muren LP, Hoyer M, Malinen E: Evaluation of adaptive radiotherapy of bladder cancer by image-based tumour control probability modelling. Acta Oncol 2010, 49:1045-1051.

21. Paddick I: A simple scoring ratio to index the conformity of radiosurgical treatment plans. Technical note. J Neurosurg 2000, 93(Suppl 3):219-222.

22. Gay HA, Niemierko A: A free program for calculating EUD-based NTCP and TCP in external beam radiotherapy. Phys Med 2007, 23:115-125.

23. Michalski JM, Gay H, Jackson A, Tucker SL, Deasy JO: Radiation dosevolume effects in radiation-induced rectal injury. Int J Radiat Oncol Biol Phys 2010, 76:S123-S129.

24. Kavanagh BD, Pan CC, Dawson LA, et al: Radiation dose-volume effects in the stomach and small bowel. Int J Radiat Oncol Biol Phys 2010, 76:S101-S107

25. Pan CC, Dawson LA, McGinn CJ, Lawrence TS, Ten Haken RK: Analysis of radiation-induced gastric and duodenal bleeds using the LymanKutcher-Burman model. Int J Radiat Oncol Biol Phys 2003, 57:S217-S218.

26. Deb P, Fielding A: Radiobiological model comparison of 3D conformal radiotherapy and IMRT plans for the treatment of prostate cancer. Australas Phys Eng Sci Med 2009, 32:51-61.

27. Henry A, Stratford J, McCarthy C, et al: X-ray Volume Imaging in Bladder Radiotherapy Verification. Int J Radiat Oncol Biol Phys 2006, 64:1174-1178.

28. Burridge N, Amer A, Marchant T, et al: Online adaptive radiotherapy of the bladder: small bowel irradiated-volume reduction. Int J Radiat Oncol Biol Phys 2006, 66:892-897.

29. McBain CA, Green MM, Stratford J, et al: Ultrasound imaging to assess inter- and intra-fraction motion during bladder radiotherapy and its potential as a verification tool. Clin Oncol (R Coll Radiol) 2009, 21:385-393.

30. Manager S, Scurr E, Huddart R, et al: Assessing intra-fraction bladder motion using cine-MRI as intial methodology for Predictive Organ Localization (POLO) in radiotherapy for Bladder Cancer. Radiother Oncol 2007, 85:207-214.

31. Bertelsen A, Hansen CR, Johansen J, Brink C: Single Arc Volumetric Modulated Arc Therapy of head and neck cancer. Radiother Oncol 2010, 95:142-148.

32. Dobler $\mathrm{B}$, Weidner K, Koelbl O: Application of volumetric modulated arc therapy (VMAT) in a dual-vendor environment. Radiat Oncol 2010, 5:95.

33. Budgell GJ, Mott JH, Logue JP, Hounsell AR: Clinical implementation of dynamic multileaf collimation for compensated bladder treatments. Radiother Oncol 2001, 59:31-38.

34. Muren LP, Redpath AT, McLaren D, et al: A concomitant tumour boost in bladder irradiation: patient suitability and the potential of intensitymodulated radiotherapy. Radiother Oncol 2006, 80:98-105.

35. Ost P, Speleers B, De Meerleer G, et al: Volumetric arc therapy and intensity-modulated radiotherapy for primary prostate radiotherapy with simultaneous integrated boost to intraprostatic lesion with 6 and $18 \mathrm{MV}$ : a planning comparison study. Int J Radiat Oncol Biol Phys 2011, 79:920-926.

36. Brenner B, Borok S, Rakowsky E, et al: Older age and second skin cancer as prognostic factors in localized malignant melanomas. Oncol Rep 2003, 10:2051-2057

37. Ruben JD, Davis S, Evans C, et al: The effect of intensity-modulated radiotherapy on radiation-induced second malignancies. Int J Radiat Oncol Biol Phys 2008, 70:1530-1536.

38. Hall EJ: Intensity-modulated radiation therapy, protons, and the risk of second cancers. Int J Radiat Oncol Biol Phys 2006, 65:1-7.
doi:10.1186/1748-717X-7-111

Cite this article as: Foroudi et al:: A dosimetric comparison of 3D conformal vs intensity modulated vs volumetric arc radiation therapy for muscle invasive bladder cancer. Radiation Oncology 2012 7:111.

\section{Submit your next manuscript to BioMed Central and take full advantage of:}

- Convenient online submission

- Thorough peer review

- No space constraints or color figure charges

- Immediate publication on acceptance

- Inclusion in PubMed, CAS, Scopus and Google Scholar

- Research which is freely available for redistribution 